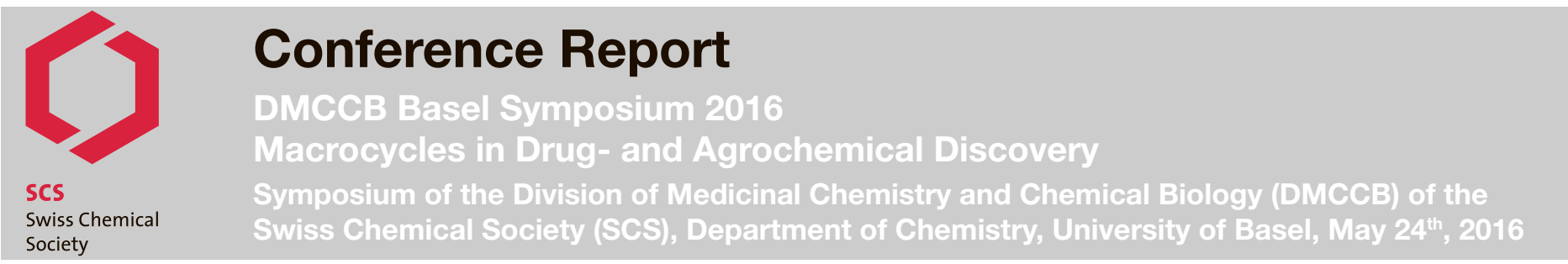

\section{Anaëlle Dumas*}

*Correspondence: Dr. A. Dumas, Institut Galien Paris-Sud, Université Paris-Sud, UMR CNRS 8612, Faculté de Pharmacie, 5 rue Jean-Baptiste Clément, 92296 Châtenay-Malabry Cedex, France. E-mail: anaelle.dumas@u-psud.fr

\section{Keywords: Agrochemistry · DMCCB · Drug · Macrocycles ·} Symposium

Macrocycles (MCs) offer a compelling approach to address challenging molecular targets such as extended binding sites and protein-protein interactions (Fig. 1). While their large surface area offer the prospect of improved biological performance compared to conventional small molecules in these roles, achieving cellular permeability and bioavailability with these scaffolds that usually fall outside definitions of conventional 'druglikeliness' is anything but trivial. How to design macrocycles with both potent target modulation and good pharmaceutical properties? And how to develop efficient and modular routes toward synthetic macrocycles? These two questions were widely addressed during the half-day symposium organized by the Division of Medicinal Chemistry and Chemical Biology (DMCCB) of the Swiss Chemical Society (SCS) in the Department of Chemistry of the University of Basel on May $24^{\text {th }}, 2016$. On this occasion, the state of the art and upcoming challenges for 'Macrocycles in Drugand Agrochemical Discovery' were methodically illustrated in a set of five lectures given by an international panel of academic and industrial experts in the field. The first part of the meeting brought the 90 attendees in a journey towards a better understanding of the key parameters for the design of biologically-performant macrocycles with two lectures discussing the topic. In the second half, two lectures introduced innovative synthetic strategies for the preparation of diversified macrocycles, followed by a talk covering their applications in agrochemistry.

\section{Cracking Macrocycle-Target Interactions}

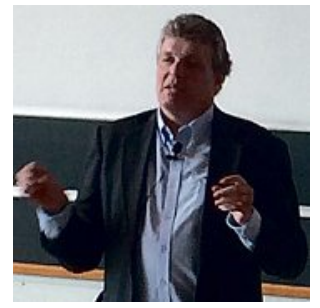

Following opening remarks by $\boldsymbol{D r}$. Yves Auberson, President of the DMCCB, Prof. Adrian Whitty from Boston University gave a lecture dedicated to 'Design considerations to bioactive macrocycles'. The first part of the talk focused on the detailed investigation of MC-Target interactions with a systematic structural analysis of MCs binding modes, performed by studying a repreAdrian Whitty sentative set of 23 macrocycle-protein complexes for which cocrystal structures have been reported. Analysing MC binding sites with FTMap, a protein mapping algorithm identifying binding hotspots of proteins, revealed that they differ from conventional drug binding sites in subtle but measurable ways. Based on these considerations, the Whitty group proposed a set of design guidelines for synthetic MCs intended as pharmaceutically useful binders/inhibitors of protein drug targets. The second part of the talk focused on MCs physicochemical parameters and guidelines to good ADMET characteristics and oral bioavailability.
The chameleonic properties of MCs that have the ability to partially bury hydrophilic or hydrophobic functionalities from their environment was invoked as a contributor to combined solubility and membrane permeability, and a proposal was advanced for how this property might be quantified and used as a parameter in molecular design (Fig. 2).

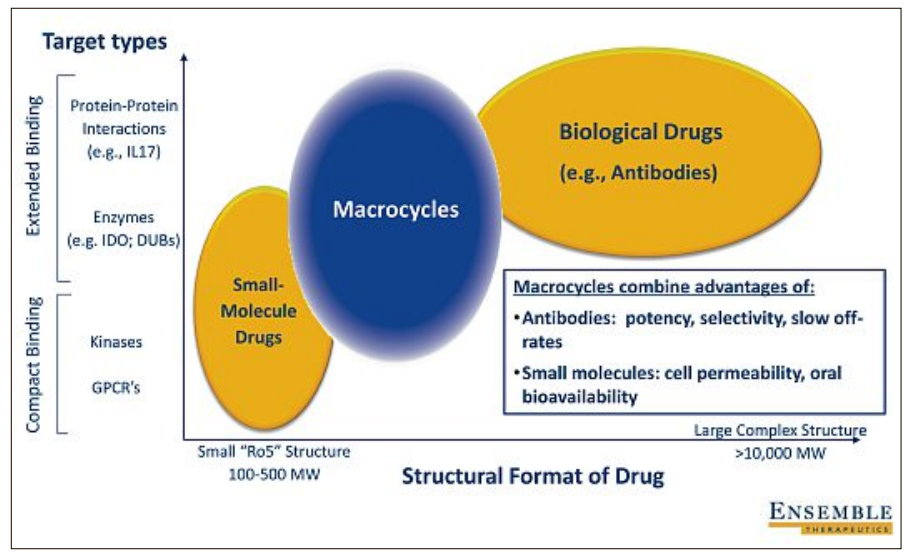

Fig. 1. Macrocycles combine advantages of antibodies and small molecules (Figure courtesy of Dr. Jeremy Duvall).

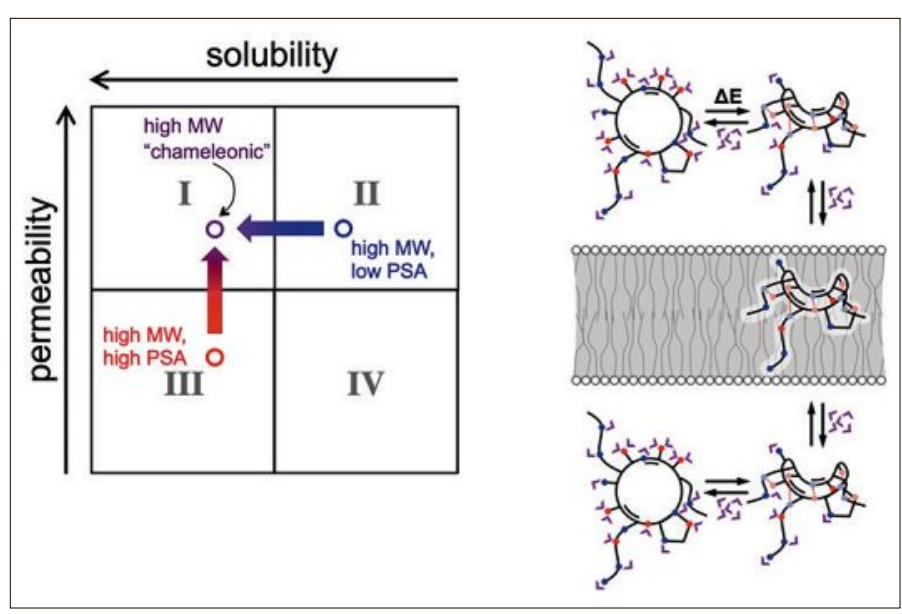

Fig. 2. Cheating the biopharmaceutical classification system with chameleons. Chameleonic properties of macrocycles can lead to compounds that are both cell-permeable and soluble (Figure courtesy of Prof. Adrian Whitty).

MCs design considerations were further discussed in a presentation entitled 'Understanding Macrocycle Permeability and Application to the Development of IDO-1 Inhibitors'. Dr. Jeremy Duvall from Ensemble Therapeutics focused on two general concepts: gaining a better understanding for the determinants of macrocyclic cell permeability and developing macrocyclic leads in the area of oncology. The first part of the talk focused on work from a collaboration between The Broad Institute of the MIT and Harvard, AstraZeneca and Uppsala University in which the team profiled more than $200 \mathrm{MCs}$ from the Diversity Oriented Synthesis collection and was able to deter- 


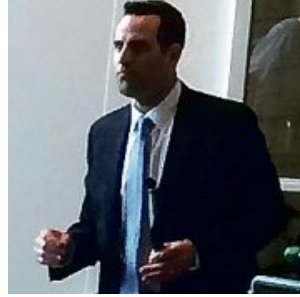

Jeremy Duvall mine the molecular, structural and conformational constraints for obtaining good cell permeability with MCs. The second half of the talk focused on work at Ensemble Therapeutics in which hits identified from their DNA-Programmed Chemistry Platform were validated and subsequently developed into leads demonstrating efficacy in the appropriate PD model. Dr. Duvall presented then two different programs - indoleamine 2,3-dioxygenase 1 (IDO) inhibitors and dimeric macrocyclic antagonists of Inhibitor of Apoptosis Protein (IAP), where findings from the first part of the talk are utilized in improving cell permeability and/or oral bioavailability.

\section{Towards Synthetic Solutions}

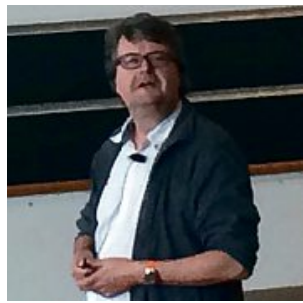

After a very lively coffee break, synthetic strategies towards MCs were on stage. Prof. Alexander Dömling, group leader at the University of Groningen introduced a highly modular approach towards synthesis of libraries of artificial MCs in a talk entitled 'Synthetic macrocycles for protein-protein interactions using multi-component reactions chemistry'. He presented a devised concept Alexander Dömling named union of MCRs (multicomponent reactions) for the fast and efficient build-up of MC: a first MCR to construct the MC backbone and a second MCR to ring-close the MC. The corresponding libraries are screened against demanding targets such as PD1-PDL1 using a number of methods including structurebased design, virtual screening, NMR-based screening and cocrystal structure analysis. Rules were discussed to increase passive membrane permeation for the MC libraries. Finally the application of MCR chemistry for the two-step construction of Gd-TEMDO, a novel macrocyclic MRI agent, was presented.

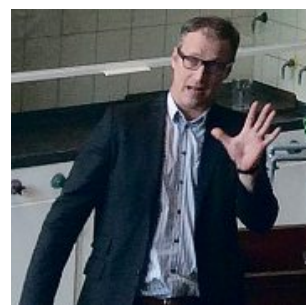

The ongoing research in Prof. Andrei Yudin's lab focusses on finding efficient routes to bioactive molecules. In his thrilling talk about 'Macrocyclization strategies based on amphoteric molecules', he described how his lab has developed amphoteric reagents that combine formally incompatible nucleophile/ electrophile combinations. Using aziridine aldehydes, the Yudin lab was able to

Andrei Yudin

show that cell-permeable peptide macrocycles can be assembled in a few simple steps. To date, they have amassed a large collection of peptide macrocycles and showed that oral bioavailability can be achieved in this class of molecules through the use of exocyclic control over intramolecular network of hydrogen bonds. In a second part, the lecture highlighted the use of Boroscan, a technology aimed at making boron-containing inhibitors of hydrolases. Given the significance of boron in contemporary drug discovery, boron-containing amphoteric molecules enable efficient construction of structurally diverse peptidomimetics containing boron atoms. Importantly, this presentation demonstrated a path toward improving cell permeability of boron-containing inhibitors. Considering the relatively low inherent toxicity of boric acid - the main metabolite of boron-containing bioactive molecules - this platform promises to streamline the synthesis of boron-containing small molecules and macrocycles.

\section{Macrocycles in the Agrochemical Field}

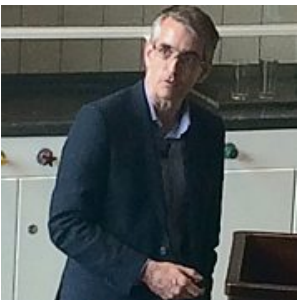

Steve Smith
For the last talk of the day, Dr. Steve Smith from Syngenta, gave us a flavour of the multiple aspects of macrocycle research in the agrochemical field with a sound talk discussing 'Macrocycles as Inspiration for Agrochemical Discovery'. In this lecture, Dr. Smith gave an overview of the high throughput in vivo approach to agrochemical discovery and the importance of natural compounds as sources of leads and products (Fig. 3). MCs form an important sub-class of natural products and Dr. Smith gave three different examples of macrocyclic synthesis from early lead discovery: 1 . Total synthesis of Macrocidin A, a macrocyclic tetramic acid; 2. Synthesis of a fungicidal natural product PF1163B and the design and efficient synthesis of a natural product like library of analogues with varying ring size. The effect of ring size on the ratio of lowest energy conformations was examined using molecular dynamics modelling; 3. Solution and solid-phase synthesis approaches to analogues of the herbicide cyclic tetrapeptide tentoxin and a discussion of physical properties limiting activity, including chameleonic polar surface area.

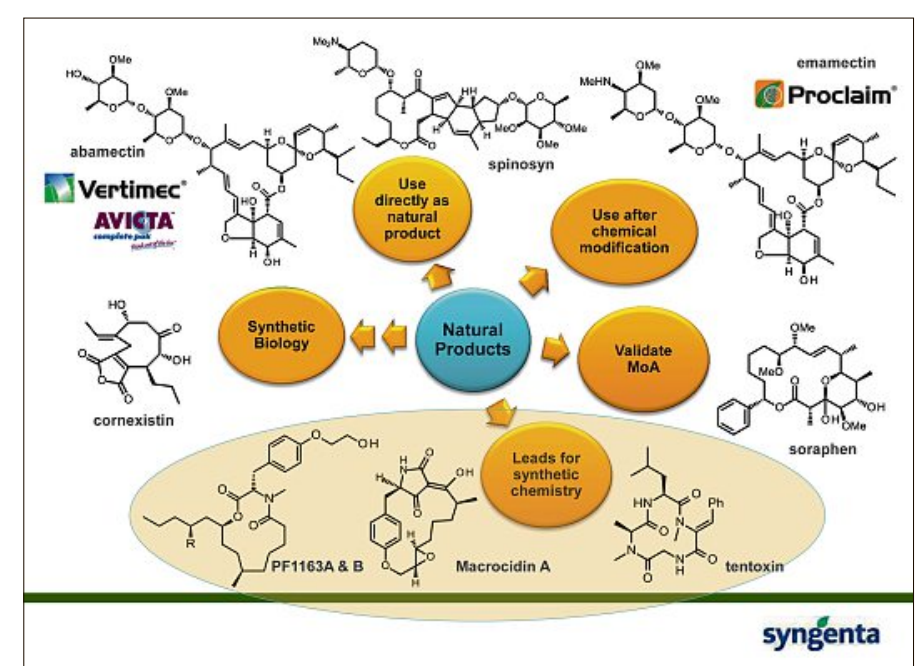

Fig. 3. Large-ring natural products in the agrochemical field (Figure courtesy of Dr. Steve Smith).

With this excellent set of diversified lectures, the DMCCB Basel Symposium 2016 proposed an extensive overview of the science of macrocycles in drug and agrochemical discovery. During these intense few hours, the audience gained a clear vision of the state of the art in macrocycle research through design, synthesis and application considerations. Before the attendees left this inspiring event, Yves Auberson expressed a special thanks to all speakers and participants, as well as the sponsors and supporters of this symposium.

Received: June 2, 2016 